\title{
FIB Tomography of Bacterial Biofilms Grown on Gold and Polystyrene
}

\author{
Jiahua $\mathrm{Gu}^{2}$, Matthew Libera ${ }^{2}$, and Tsengming $\mathrm{Chou}^{1}$ \\ 1. Laboratory of Multiscale Imaging, Stevens Institute of Technology, Hoboken, NJ USA \\ 2. Dept. of Chemical Engr. \& Materials Science, Stevens Institute of Technology, Hoboken, NJ USA
}

Bacterial biofilms are three-dimensional communities of bacteria distributed in a highly hydrated extracellular matrix (ECM) (Fig.1). They have structures over multiple length scales ranging from nanoscale to macroscale. Following standard EM sample-preparation protocols of embedding cellular samples [1], we can fix, stain, and embed biofilms while maintaining the close to their native state. This enables us to study their three-dimensional structure using focused ion beam (FIB) tomography [2].

Staphylococcus aureus (S. aureus; ATCC-12600) was cultured in a polystyrene (PS) culture dish where half of the dish was sputter coated with $\sim 5 \mathrm{~nm}$ of gold (Au). Both sides were subsequently subjected to identical bacterial inoculation $\left(1 \times 10^{9} \mathrm{CFU} / \mathrm{ml}\right)$, culture (tryptic soy broth, replace with fresh medium six times over $48 \mathrm{~h}$ period), and specimen preparation processes. The stained biofilm appears darker after embedding on the Au side (Fig. 2(a)). Small blocks were cut from each side and then trimmed and faced using glass knives in a Leica Ultracut S microtome. A Zeiss Auriga 40 FIB-SEM was used to slice and image the samples. The energy-selective-backscattering detector (EsB) was used for imaging. An Atlas 3D (Fibics, Inc.) was used to control the FIB-SEM while collecting the data. The Fibics system compensates for the focus and image shift during data collection. Image data were collected over milling periods of approximately $12 \mathrm{~h}$. A typical dataset contained 1,000 images collected from a specimen volume of $30 \mu \mathrm{m} \times 20 \mu \mathrm{m} \times 20 \mu \mathrm{m}$ with a $10 \mathrm{~nm} \times 10 \mathrm{~nm}$ image pixel resolution and $20 \mathrm{~nm}$ slice thickness. Images were processed and analysed using the Avizo Fire program (FEI Software).

Fig. 2(b) and 2(c) show FIB cross-sections of the biofilm cultured on Au and PS. The Au thin film corresponds to the bright line near the bottom of fig. 2(b). The bacteria only cover part of the surface and grow upward to form the biofilm. With little scattering contrast between the PS substrate and the embedding epoxy, the biofilm-PS substrate is barely visible in Fig. 2(c), and a dashed line marker is included to indicate this interface more clearly. Note that a significant fraction of the biofilm-substrate interface is not covered by bacteria, which suggests that biofilm attachment can be mediated by only a few attachment events. Fig. 3 shows a 3D image reconstruction of the biofilm cultured on Au. Table 1 summarizes some characteristics of the biofilms grown on each substrate. Importantly, the number of bacteria per unit area at the biofilm-substrate interface is about the same, but the volume fraction of bacteria within the biofilm are higher for the biofilm grown on PS relative to that grown on Au. These results suggest that the nature of the substrate can influence the mesoscale structure of an entire biofilm, much of which has no direct contact with the substrate.

\section{References:}

[1] John J. Bozzola, Ch.1 in "Methods in Molecular Biology", vol. 369, ed. J. Kuo, Humana Press, Totowa, NJ, p.1-18

[2] A. Bushby et al, Nature Protocols, vol. 6, no. 6 (2011), p.845 - 858

[3] This project was supported by the U.S. Army Research Office via grant W911NF-12-1-0331 using microscope resources partially funded by the National Science Foundation via Grant DMR-0922522 


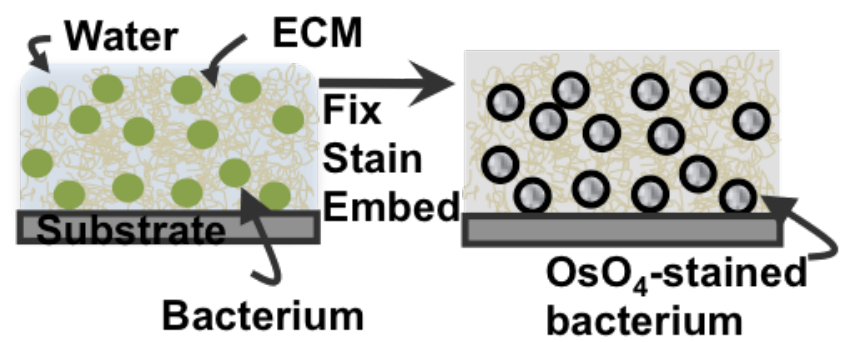

Fig. 1 Schematic of a biofilm grown on a solid surface. Each bacterium is approximately $1 \mu \mathrm{m}$ in diameter. Fixed/dried/embedded samples were used for FIB-SEM study.

\begin{tabular}{|l|l|l|} 
& Au & PS \\
\hline $\begin{array}{l}\text { Volume fraction of } \\
\text { bacteria }\end{array}$ & $\mathbf{3 2 \%}$ & $\mathbf{4 3 \%}$ \\
\hline $\begin{array}{l}\text { Number of bacteria } \\
\text { at interface }\left(\# / \mu \mathrm{m}^{2}\right)\end{array}$ & $\mathbf{0 . 3 8}$ & $\mathbf{0 . 3 9}$ \\
\hline
\end{tabular}

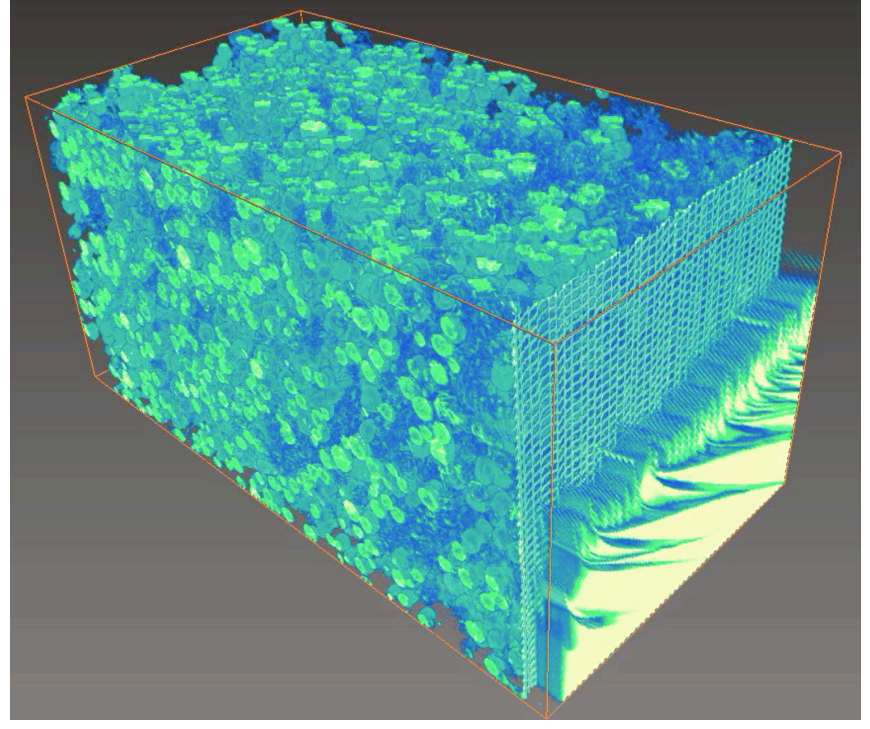

Fig. 3 3D image reconstruction of the biofilm grown on Au. Voxel size is $10 \mathrm{~nm}$ x $10 \mathrm{~nm} \times$ $20 \mathrm{~nm}$ and the data cube volume is $30 \mu \mathrm{m} \times 20$ $\mu \mathrm{m} \times 19.5 \mu \mathrm{m}$.

Table 1. Characteristics of the biofilms grown on Au and PS .

(a)

(b)

(c)

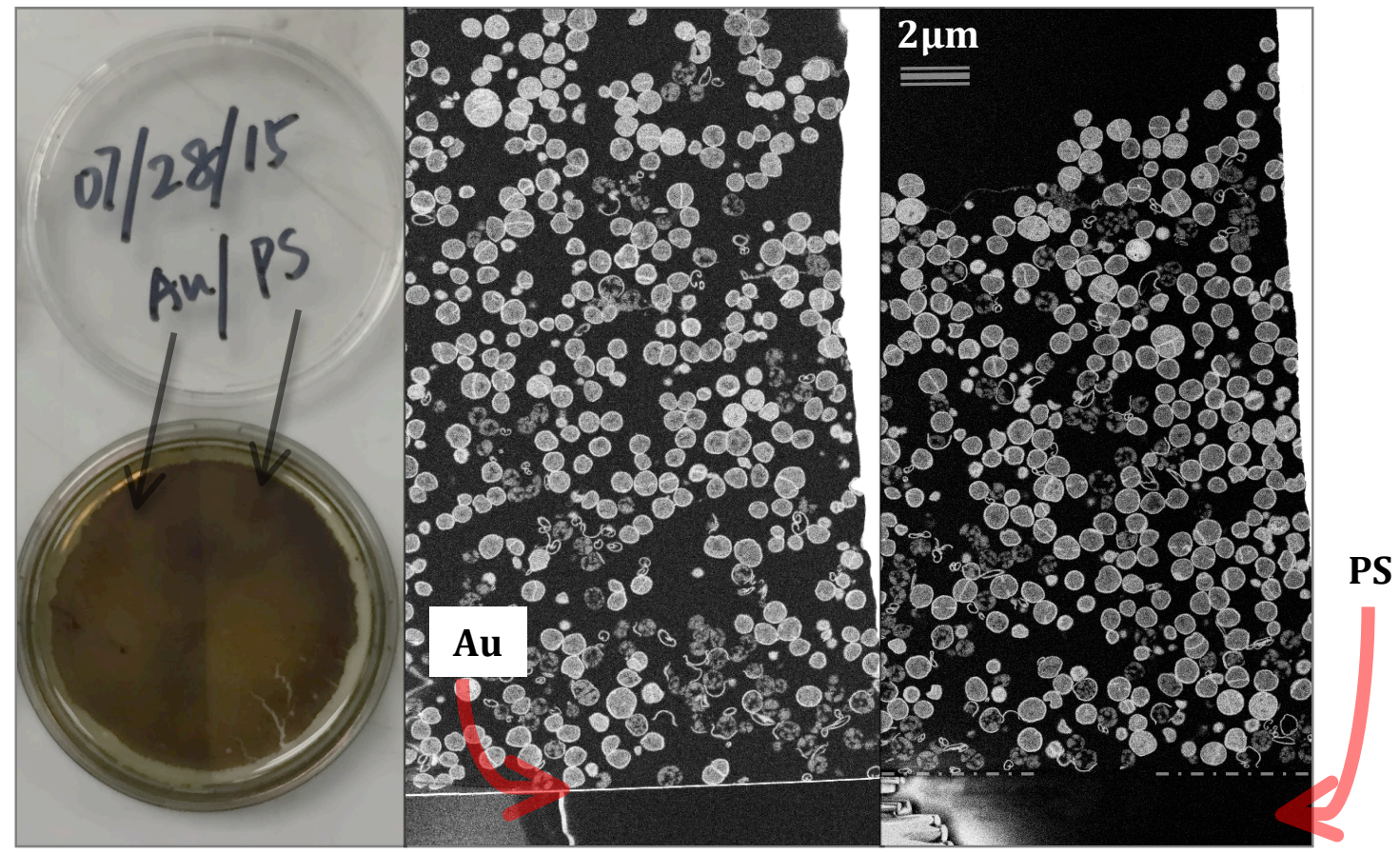

Fig. 2 (a) Bacterial biofilm grown in a PS culture dish where half of the dish was sputter coated with $\sim 5 \mathrm{~nm}$ of Au. (b) FIB cross-section of the biofilm grown on $\mathrm{Au}(\mathrm{c}) \mathrm{FIB}$ cross-section of the biofilm grown on PS. 